\title{
A MEAN-FIELD GAME APPROACH TO PRICE FORMATION
}

\author{
DIOGO A. GOMES AND JOÃO SAÚDE
}

\begin{abstract}
Here, we introduce a price-formation model where a large number of small players can store and trade a commodity such as electricity. Our model is a constrained mean-field game (MFG) where the price is a Lagrange multiplier for the supply vs. demand balance condition. We establish the existence of a unique solution using a fixed-point argument. In particular, we show that the price is well-defined, and it is a Lipschitz function of time. Then, we study linear-quadratic models that can be solved explicitly and compare our model with real data.
\end{abstract}

\section{INTRODUCTION}

The mean-field game (MFG) framework [27, 28, 33, 34] models systems with many rational players (see, e.g., the surveys [23] and [24]). Here, we are interested in the price formation in electricity markets. In our model, a large number of agents own storage devices that can be charged and later supply the grid with electricity. Agents seek to maximize profit by trading electricity at a price $\varpi(t)$, which is set by a supply versus demand balance condition.

With the advent of electric cars, a large number of network-connected batteries are already available, and their number is only likely to increase. Moreover, energy can be stored as heat or cold, using space or water heaters and air-conditioning units [30, 31, 29]. With new small network-capable devices, appliances can be connected to the grid and use smart algorithms to control their energy usage. These algorithms can balance supply and demand and, thus, are particularly relevant when combined with solar and wind energy production, where power demand seldom matches production. The original motivation of this work was the study of price formation in electricity markets. However, our model describes as well the price formation in a single-commodity market with exogenous supply. Nevertheless, for definiteness, we keep the electricity model as our main application.

Price formation models were some of the first MFG models [35]. This line of research was pursued by several authors, see [6, 5, 40, 3, 4, 32, 19] and the monograph [20]. Some of these models are formulated as free boundary problems [6, 5]; others as a load control problem [39, 38]. For example, using mean-field control and MFG, the load-control problem through switching on and off space heaters was studied in $[30,31,29]$. Previous authors addressed the price issue by assuming that the demand is a given function of the price [36] or that the price is a given function of the demand, see [11], [10], [12], [13], and [26]. In particular, in [13], the authors use

Date: February 29, 2020.

2010 Mathematics Subject Classification. 91A13, 91A10, 49M30.

Key words and phrases. Mean-field games; Price formation; Monotonicity methods.

D. Gomes was partially supported by KAUST baseline funds and KAUST OSR-CRG20173452. J. Saúde was partially supported by FCT/Portugal through the CMU-Portugal Program. 
a price function to study mean-field equilibrium in electricity markets in a setting that is similar to ours.

Here, we pursue a different approach: often, in economic models, prices of goods and services are determined by the balance between supply and demand rather than by a given function of the supply. Therefore, the price as a function of the supply or demand is not known a priori and a key unknown in the problem. This observation motivated the approach in [19], where price arises from supply versus demand constraints. However, that model is more complex than the one discussed here and was only studied from a numerical perspective. Thus, mathematical issues such as the existence and uniqueness of a price, the well-posedness of the model, and the convergence of numerical methods were left unanswered and are settled here.

Our model comprises three quantities of interest: a price $\varpi \in C([0, T])$, a value function $u \in C(\mathbb{R} \times[0, T])$, and a path describing the statistical distribution of the agents, $m \in C([0, T], \mathcal{P})$, where $\mathcal{P}$ is the set of probability measures in $\mathbb{R}$ with bounded first moment, endowed with the 1-Wasserstein distance. These quantities are determined by the following problem.

Problem 1. Given $\epsilon \geq 0$, a Hamiltonian, $H: \mathbb{R} \times \mathbb{R} \rightarrow \mathbb{R}, H \in C^{\infty}$, an energy production rate $\mathbf{Q}:[0, T] \rightarrow \mathbb{R}, \mathbf{Q} \in C^{\infty}([0, T])$, a terminal cost $\bar{u}: \mathbb{R} \rightarrow \mathbb{R}$, $\bar{u} \in C^{\infty}(\mathbb{R})$ and an initial probability distribution $\bar{m} \in \mathcal{P} \cap C_{c}^{\infty}(\mathbb{R})$, find $u: \mathbb{R} \times$ $[0, T] \rightarrow \mathbb{R}, m \in C([0, T], \mathcal{P})$, and $\varpi:[0, T] \rightarrow \mathbb{R}$ solving

$$
\left\{\begin{array}{l}
-u_{t}+H\left(x, \varpi(t)+u_{x}\right)=\epsilon u_{x x} \\
m_{t}-\left(D_{p} H\left(x, \varpi(t)+u_{x}\right) m\right)_{x}=\epsilon m_{x x} \\
\int_{\mathbb{R}} D_{p} H\left(x, \varpi(t)+u_{x}\right) d m=-\mathbf{Q}(t),
\end{array}\right.
$$

and satisfying the initial-terminal conditions

$$
\left\{\begin{array}{l}
u(x, T)=\bar{u}(x), \\
m(x, 0)=\bar{m}(x) .
\end{array}\right.
$$

In the previous problem, $x \in \mathbb{R}$ represents the state of a typical agent; that is, the energy stored by the agent. The function $u(x, t)$ is the value function for an agent whose charge is $x$ at time $t$. The Hamiltonian, $H: \mathbb{R} \times \mathbb{R} \rightarrow \mathbb{R}$ is determined by the optimization problem that each agent seeks to solve, as described in Section 2. We require $u$ to be a viscosity of the first equation in (1). However, if $\epsilon>0$, parabolic regularity theory gives additional regularity for $u$. For each $t \in[0, T], m$ determines the distribution of the energy storage of the agents. Here, we assume that $m$ is a weak solution of the second equation in (1); that is, for every $\psi \in C_{c}^{2}(\mathbb{R} \times[0, T])$, we have

$$
\begin{aligned}
\int_{0}^{T} & \int_{\mathbb{R}}\left(\psi_{t}+\psi_{x} D_{p} H\left(x, \varpi+u_{x}\right)-\epsilon \psi_{x x}\right) m d x d t \\
= & \int_{\mathbb{R}} \psi(x, T) m(x, T) d x-\int_{\mathbb{R}} \psi(x, 0) \bar{m}(x) d x
\end{aligned}
$$

Thus, the second equation in (1) is well defined for $m \in C([0, T], \mathcal{P})$. However, when $\epsilon>0, m$ is absolutely continuous, see Remark 6 . This may not be the case when $\epsilon=0$. In this case, integral expressions such as $\int_{\mathbb{R}} f(x) m(x, t) d x$ should be understood as $\int_{\mathbb{R}} f(x) m(d x, t)$ or simply $\int_{\mathbb{R}} f d m(t)$. 
The fact that $\bar{m}$ has compact support simplifies the analysis in various points. For example, automatically $\bar{m}$ has finite second-moments, as required by Proposition 4. With a more detailed analysis, it would be certainly possible to consider a larger class of functions for $\bar{m}$.

The parameter $\epsilon$ corresponds to random fluctuation in the storage of the agents. Finally, the spot price, $\varpi(t)$, is selected so that the total energy used balances the supply, $\mathbf{Q}(t)$, the condition imposed by the last equation in (1).

In the current model, agents have a time horizon $T>0$, and, at time $T$, they pay a terminal cost, $\bar{u}(x)$, that depends on their state at the terminal time. For example, agents may prefer to have the batteries fully charged at the end of the day. Moreover, the initial distribution of agents, $\bar{m}$, is known. These two facts are encoded in the initial-terminal boundary conditions, (2). This model can easily be modified to address periodic in time boundary conditions and the infinite horizon discounted problem.

First, in Section 2, we present a derivation of our model and examine some of its mathematical properties. Then, after a brief discussion of the main assumptions, in Section 3, we prove our main result given by the following theorem.

Theorem 1. Suppose that Assumptions 1-5 (see Section 3) hold. Then, there exists a solution $(u, m, \varpi)$ of Problem 1 where $u$ is a viscosity solution of the first equation, Lipschitz and semiconcave in $x$, and differentiable almost everywhere with respect to $m, m \in C([0, T], \mathcal{P})$, and $\varpi \in W^{1,1}$ Moreover, if $\epsilon>0$ this solution is unique.

If $\epsilon=0$ and Assumption 6 holds, then there is a unique solution $(u, m, \varpi)$. Moreover, $u$ is differentiable in $x$ for every $x$, and $u_{x x}$ and $m$ are bounded.

The proof of the previous theorem is based on a fixed-point argument. For that, we need to establish the boundedness and continuity of various maps. First, in Propositions 1, 2 and 3, we study properties of solution of the Hamilton-Jacobi equation as a function of the price. Next, in Propositions 4, we examine the dependence of the solution of the Fokker-Planck equation. The analysis here, is quite similar to arguments in MFG theory, see, for example chapters 3 and 4 in [23]. However, particular care has to be taken to establish estimates that are uniform in the price. Subsequently, we establish a differential equation satisfied by the price, equation (20), that is used in Proposition 6 to establish the boundedness of the price in $W^{1, \infty}$. This approach is specific to this price problem and, we believe, has no analog in the literature. These results are combined at the end of Section 4 to prove the existence part of the theorem.

Remark 1. If $\epsilon>0$, the regularity of the solutions can be improved using parabolic regularity, using, for example, the estimates similar to the ones in [21] or [22] and Remark 6. Here, however, we do not pursue this direction. A detailed account of a priori estimates for parabolic problems can be found in [23].

There are two main contributions of this paper. First, is the existence part of the preceding theorem which is proved in Section 4 using a fixed-point argument. The key step is establishing an ordinary differential equation satisfied by the price, $\varpi$. Using this equation, we obtain Lipschitz bounds and then apply Schauder's fixed-point theorem. To prove the uniqueness part of the theorem, we use the monotonicity method. This is achieved in Section 5 where we identify a new monotonicity structure for mean-field games with constraints. Finally, we discuss 
linear-quadratic models, that can be solved explicitly and compare our model with the ones in [12]. Although linear-quadratic models have been considered previously in the literature, see for example [23] for a brief survey of existing results, to the best of our knowledge, this is the first case where a linear-quadratic mean-field game is coupled with an equilibrium condition. Our results suggest that a price determined by a supply versus demand condition may help stabilize the oscillations of the price in particular in peak-demand situations.

Notation. $C^{k}$ or $C^{k}\left(\mathbb{R}^{d}\right)$ is the set of $k$ times continuously differentiable functions in $\mathbb{R}^{d}$. $C_{c}^{k}$ is the set of compactly supported $C^{k}$ functions. Finally, $W^{1, p}$ with $1 \leq k \leq+\infty$, is the Sobolev space of weakly differentiable functions in $\mathbb{R}^{d}$, endowed with the norm $\|u\|_{W^{1, p}}=\|u\|_{L^{p}}+\|D u\|_{L^{p}}$.

\section{A MEAN-FIELD MODEL FOR PRICE FORMATION}

Here, we present the derivation of our price model. To simplify the discussion, we examine the deterministic case, $\epsilon=0$. We consider an electricity grid connecting consumers to producers of energy. In our model, each consumer has a storage device connected to the network, for example, an electric car battery. We assume that all devices are similar. Consumers trade electricity, charging the batteries when the price is low and selling electricity to the market when the price is high. A typical consumer has a battery whose charge at time $t \in[0, T]$ is $\mathbf{x}(t)$. This charge changes according to an energy flow rate, the control variable selected by each consumer, which is a bounded measurable function of $\alpha:[0, T] \rightarrow A$, where $A \subset \mathbb{R}$. Positive values of $\alpha$ correspond to buying energy from the grid, and negative values to selling to the grid. Accordingly, each consumer charge, $\mathbf{x}$, changes according to the dynamics:

$$
\dot{\mathbf{x}}(t)=\alpha(t) .
$$

Each consumer seeks to select $\alpha$ to minimize its cost, thus maximizing profit. In principle, we could consider more complex dynamics, for example

$$
\dot{\mathbf{x}}(t)=q(\mathbf{x}, \alpha(t)) .
$$

However, this would require different methods to be studied, for example, Propositions 1 and 2 use the special form of (32).

Th cost of the players is determined by a terminal cost and by the integral of the running cost, $\ell(\alpha, x, t)$, where $\alpha(t)$ is the energy traded with the electricity grid at time $t$, and $\ell$ depends in time through $\varpi(t)$, the spot electricity price and is of the form

$$
\ell(\alpha, x, t)=\ell_{0}(\alpha, x)+\varpi(t) \alpha .
$$

In the preceding expression, the term $\varpi(t) \alpha(t)$ is the instantaneous cost corresponding to a charging current $\alpha(t)$. The current (or more precisely power), $\alpha$ is measured in Watt, $\mathrm{W}$, and the price, $\varpi$, in $\$ \mathrm{~W}^{-1} s^{-1}$. The function $\ell_{0}$ accounts for non-linear effects of the current usage, for example, battery wear and tear, and for state preferences. For example, we often take

$$
\ell_{0}(\alpha, x, t)=\frac{c}{2} \alpha^{2}+V(x),
$$


where $c$ is a constant that accounts for the battery's wear off, typically given in $\$ \mathrm{~W}^{-2} s^{-1}$, and $V(x)$ is a potential that takes into account battery constraints and charge preferences. The singular case where

$$
V(x)=\left\{\begin{array}{lr}
0 & \text { if } 0 \leq x \leq 1 \\
+\infty & \text { otherwise }
\end{array}\right.
$$

corresponds to the case where the battery charges satisfies $0 \leq x \leq 1$. To avoid singularities, we work with smooth potentials growing as $x \rightarrow \pm \infty$; this corresponds to a penalty on the battery charge rather than a hard constraint. The nonlinear term, $\frac{c}{2} \alpha^{2}(t)$, models battery wear and tear, which is large in high-current regimes. The particular quadratic form in (5) simplifies the mathematical treatment. However, it can be replaced by a convex function of $\alpha$ without any major change in the discussion.

Each consumer minimizes the functional

$$
J(x, t, \alpha)=\int_{t}^{T} \ell(\alpha(s), \mathbf{x}(t), t) d s+\bar{u}(\mathbf{x}(T)),
$$

where $\bar{u}$ is the terminal cost and $\alpha \in \mathcal{A}_{t}$, where $\mathcal{A}_{t}$ is the set of bounded measurable functions $\alpha:[t, T] \rightarrow A$.

The value function, $u$, is the infimum of $J$ over all controls in $\mathcal{A}_{t}$; that is,

$$
u(x, t)=\inf _{\alpha \in \mathcal{A}_{t}} J(x, t, \alpha) .
$$

The Hamiltonian, $H$, for the preceding control problem is

$$
H(x, p)=\sup _{a \in A}\left(-p a-\ell_{0}(x, a)\right) .
$$

For example, for $\ell_{0}$ as in (5), we have

$$
H(x, p)=\frac{p^{2}}{2 c}-V(x) .
$$

From standard optimal control theory, $u$ is a viscosity solution (see [2]) of the Hamilton-Jacobi equation

$$
\left\{\begin{array}{l}
-u_{t}+H\left(x, \varpi(t)+u_{x}\right)=0 \\
u(x, T)=\bar{u}(x) .
\end{array}\right.
$$

For $\ell_{0}$ as in (5), the prior equation becomes

$$
-u_{t}+\frac{1}{2 c}\left(u_{x}+\varpi(t)\right)^{2}-V(x)=0 .
$$

Finally, at points of differentiability of $u$, the optimal control is given in feedback form by

$$
\alpha^{*}(x, t)=-D_{p} H\left(x, \varpi(t)+u_{x}(\mathbf{x}(t), t)\right) .
$$

The associated transport equation is the adjoint of the linearized Hamilton-Jacobi equation:

$$
\left\{\begin{array}{l}
m_{t}-\left(D_{p} H\left(x, u_{x}+\varpi(t)\right) m\right)_{x}=0, \\
m(x, 0)=\bar{m}(x),
\end{array}\right.
$$

where $\bar{m}$ is the initial distribution of the agents. 
Taking $\ell_{0}$ as in (5), the transport equation above becomes

$$
m_{t}-\frac{1}{c}\left(m\left(\varpi+u_{x}\right)\right)_{x}=0 .
$$

Finally, we fix an energy production function $\mathbf{Q}(t)$ and require that the production balances demand. Mathematically, this constraint corresponds to the identity

$$
\int_{\mathbb{R}} \alpha^{*}(t) m(x, t) d x=\mathbf{Q}(t)
$$

that is,

$$
\int_{\mathbb{R}} D_{p} H\left(x, u_{x}+\varpi(t)\right) m(x, t) d x=-\mathbf{Q}(t) .
$$

This foregoing equality is the balance equation that forces the consumed energy to match the production; this constraint determines the price, $\varpi(t)$.

Combining (7), (8) and (9), we obtain (1) with $\epsilon=0$ and the initial-terminal conditions (2).

Now, we consider the case where the agents are subject to independent random consumption; that is, $\epsilon>0$. Let $(\Omega, \mathcal{F}, P)$ be a probability space, where $\Omega$ is a sample space, $\mathcal{F}$ a $\sigma$-algebra on $\Omega$ and $P$ a probability measure. Let $W_{t}$ be a Brownian motion on $\Omega$ and $\left\{\mathcal{F}_{t}\right\}_{t \geq 0}$ the associated filtration. In this case, we model the agent's motion by the stochastic differential equation

$$
d \mathbf{x}(t)=\alpha(t) d t+\sqrt{2 \epsilon} d W_{t},
$$

where the control, $\alpha$, is a bounded progressively measurable real-valued process. Following the previous steps and using standard arguments in stochastic optimal control, we arrive again at (1).

\section{Main Assumptions}

We begin by discussing our main assumptions. First, we suppose that $H$ is the Legendre transform of a Lagrangian that is the sum of an "energy flow cost", $\ell_{0}(\alpha)$, and a "charge preference cost", $V(x)$, as follows.

Assumption 1. The Hamiltonian $H$ is the Legendre transform of a convex Lagrangian:

$$
H(x, p)=\sup _{\alpha \in \mathbb{R}}-p \alpha-\ell_{0}(\alpha)-V(x),
$$

where $\ell_{0} \in C^{2}(\mathbb{R})$ is a uniformly convex function (that is, for all $\alpha, D_{\alpha^{2}}^{2} \ell_{0}(\alpha) \geq$ $\kappa>0$, for some positive real $\kappa$ ) and $V \in C^{2}(\mathbb{R})$ is bounded from below.

Remark 2. The preceding assumption is natural in the context of price models as the trading costs $\ell_{0}(\alpha)$ can often be considered independent of the current state of the agent $(x)$ and, thus, of the storage/preference costs $V(x)$. From the mathematical point of view, this structure simplifies some arguments; for example, Propositions 1 and 2. However, it may be possible to work under more general assumptions.

Remark 3. By preceding hypothesis, $H$ is given by a Legendre transform, Thus, the map $p \mapsto H(x, p)$ is convex. Further, because $\ell_{0}$ is unifomly convex, we have that $\ell_{0}$ is coercive. Thus, it follows that $p \mapsto H(x, p)$ is strictly convex; that is $D_{p p}^{2} H(x, p)>0$, see [14]. Moreover, the Hamiltonian in (10) can be written as

$$
H(x, p)=H_{0}(p)-V(x) .
$$


Thus,

$$
D_{x p}^{2} H(x, p)=0
$$

for all $x, p \in \mathbb{R}$.

To obtain a fixed point, we need several a priori estimates. These depend on convexity and regularity properties of the data. The following two assumptions lay out our requirements on the potential, $V$.

Assumption 2. The potential $V$ in (10) and the terminal data $\bar{u}$ are globally Lipschitz.

Assumption 3. The potential $V$ in (10) and the terminal data $\bar{u}$ satisfy

$$
\left|D_{x x}^{2} V\right| \leq C, \quad\left|D_{x x}^{2} \bar{u}\right| \leq C
$$

for some positive constant $C$.

Next, we state an additional regularity for the initial-terminal data that is used to prove second-order estimates.

Assumption 4. There exists a constant, $C>0$, such that

$$
\left|D_{x x}^{2} \bar{m}\right|,\left|D_{x x}^{2} \bar{u}\right| \leq C .
$$

The next two assumptions are used to ensure the solvability of the demandsupply relation; that is, given $\mathbf{Q}$ that we can determine a suitable price.

Assumption 5. There exists $\theta>0$ such that

$$
D_{p p}^{2} H(x, p)>\theta
$$

for all $x, p \in \mathbb{R}$. In addition, there exists $C>0$ such that

$$
\left|D_{p p p}^{3} H\right| \leq C .
$$

Remark 4. Using (11) in Remark 3, the preceding assumption combined with Assumption 1 implies that the function $p \mapsto D_{p} H(p, x)$ is strictly increasing with stricly positive derivative (by the uniform convexity), therefore, and

$$
\lim _{p \rightarrow-\infty} D_{p} H(p, x)=-\infty \quad \lim _{p \rightarrow+\infty} D_{p} H(p, x)=+\infty,
$$

uniformly in $x$.

Remark 5. The uniform convexity of $\ell_{0}$ in Assumption 1 gives an upper bound for $D_{p p}^{2} H$. Thus, Assumption 1 and 5 imply

$$
\left|D_{p p}^{2} H(x, p)\right| \leq C
$$

for all $x, p \in \mathbb{R}$.

The following hypothesis gives regularity and uniqueness of solutions in the firstorder case.

Assumption 6. The potential, $V$, and the terminal cost, $\bar{u}$, are convex. 


\section{EXISTEnCE OF A SOLUtion}

Here, we establish the existence of a solution for the price model, (1), using a fixed-point argument on $\varpi$. In the following two propositions, we examine the Hamilton-Jacobi equation

$$
\left\{\begin{array}{l}
-u_{t}+H\left(x, \varpi+u_{x}\right)=\epsilon u_{x x} \\
u(x, T)=\bar{u}(x)
\end{array}\right.
$$

Both proofs rely on the structural properties implied by Assumption 1: the Lagrangian corresponding to (12) is linear in the price and the only dependence the state, $x$, is through the potential, and, thus independent on the control. First, using Assumption 2, we prove the Lipschitz continuity of $u$. Next, using Assumption 3, we obtain the semiconcavity of $u$. The proofs follow standard arguments in optimal control theory. However, we present them here to make it evident that the Lipschitz and semiconcavity constants are uniform in $\varpi$ and $\epsilon$, both essential points in our argument.

The proofs of Propositions 1 and 2 rely on the fact that the value function is the unique viscosity solution of (12) with the corresponding, see for example [14], chapter 10 .

Proposition 1. Consider the setting of Problem 1 and suppose that Assumptions 1 and 2 hold. Let $u$ solve (12). Then, $u(x, t)$ is locally bounded and the map $x \mapsto u(x, t)$ is Lipschitz for $0 \leq t \leq T$. Moreover, the Lipschitz bound on $u$ does not depend on $\varpi$ nor on $\epsilon$.

Proof. The proof follows from the representation of $u$ as a solution to a stochastic control problem (or deterministic if $\epsilon=0$ ). We fix a filtered probability space $\left(\Omega, \mathcal{F}_{t}, P\right)$ that supports a one-dimensional Brownian motion $W_{t}$. Then,

$$
u(x, t)=\inf E\left[\int_{t}^{T} \ell_{0}(\alpha)+\varpi \alpha+V(\mathbf{x}) d s+\bar{u}(\mathbf{x}(T))\right],
$$

where the infimum is taken over bounded progressively measurable controls $\alpha$ : $[t, T] \rightarrow \mathbb{R}$ and $\mathbf{x}$ solves the stochastic differential equation

$$
d \mathbf{x}=\alpha d t+\sqrt{2 \epsilon} d W_{t} .
$$

To prove local boundedness, we use the sub-optimal control $\alpha \equiv 0$ to get an upper bound, and the fact that $V$ is bounded by below to obtain the lower bound. We observe, however, that the lower bound depends on bounds on $\varpi$.

Under our assumptions, there exists an optimal control $\alpha^{*}$, see the discussion in [18]. However, the argument below, does not require this, it is enough to work with $\delta$-optimal controls, that is, controls that satisfy

$$
u(x, t) \geq E\left[\int_{t}^{T} \ell_{0}\left(\alpha^{\delta}\right)+\varpi \alpha^{*}+V\left(\mathbf{x}^{\delta}\right) d s+\bar{u}\left(\mathbf{x}(T)^{\delta}\right)\right]-\delta .
$$

and let $\delta \rightarrow 0$.

Then, we fix an optimal control, $\alpha^{*}$, for $(x, t)$; that is,

$$
u(x, t)=E\left[\int_{t}^{T} \ell_{0}\left(\alpha^{*}\right)+\varpi \alpha^{*}+V\left(\mathbf{x}^{*}\right) d s+\bar{u}\left(\mathbf{x}(T)^{*}\right)\right] .
$$


Then, for any $h \in \mathbb{R}$, we have

$$
u(x+h, t) \leq E\left[\int_{t}^{T} \ell_{0}\left(\alpha^{*}\right)+\varpi \alpha^{*}+V\left(\mathbf{x}^{*}+h\right) d s+\bar{u}\left(\mathbf{x}(T)^{*}+h\right)\right],
$$

from which the Lipschitz bound follows. Note that this Lipschitz bound does not depend on $\varpi$, only on $T$ and on the Lipschitz estimates for $V$ and $\bar{u}$.

Proposition 2. Consider the setting of Problem 1 and suppose that Assumptions 1 and 3 hold. Then, $x \mapsto u(x, t)$ is semiconcave with a semiconcavity constant that does not depend on $\epsilon$ nor on $\varpi$.

Proof. As before, we fix an optimal control $\alpha^{*}$ for $(x, t)$; that is,

$$
u(x, t)=E\left[\int_{t}^{T} \ell_{0}\left(\alpha^{*}\right)+\varpi \alpha^{*}+V\left(\mathbf{x}^{*}\right) d s+\bar{u}\left(\mathbf{x}(T)^{*}\right)\right] .
$$

Then, for any $h \in \mathbb{R}$, we have

$$
u(x \pm h, t) \leq E\left[\int_{t}^{T} \ell_{0}\left(\alpha^{*}\right)+\varpi \alpha^{*}+V\left(\mathbf{x}^{*} \pm h\right) d s+\bar{u}\left(\mathbf{x}(T)^{*} \pm h\right)\right] .
$$

Therefore,

$$
u(x+h, t)-2 u(x, t)+u(x-h, t) \leq C h^{2} .
$$

Note that $C$ does not depend on $\varpi$, only on $T$ and on the semiconcavity estimates for $V$ and $\bar{u}$.

We have the following stability properties for the solutions of (12).

Proposition 3. Consider the setting of Problem 1 and suppose that Assumptions 1-3 hold. Suppose that $\varpi_{n} \rightarrow \varpi$ uniformly on $[0, T]$, then $u^{n} \rightarrow u$ locally uniformly and $u_{x}^{n} \rightarrow u_{x}$ almost everywhere.

Proof. The local uniform convergence of $u^{n}$ follows from the stability of viscosity solutions, see [14], chapter 10. Because $u^{n}$ is semiconcave and converges uniformly to $u, u_{x}^{n} \rightarrow u_{x}$ almost everywhere. This is so because $u_{x}^{n}$ is a subdifferential of $u^{n}$ and, therefore, any sublimit is a subdifferential of $u$. Since $u$ is differentiable almost everywhere, this limit is unique for almost every $x$. Therefore, $u^{n}$ is semiconcave and converges uniformly to $u$.

Now, we examine the Fokker-Planck equation.

$$
\left\{\begin{array}{l}
m_{t}-\operatorname{div}\left(m D_{p} H\left(x, \varpi+u_{x}\right)\right)=\epsilon \Delta m, \\
m(x, 0)=\bar{m}(x) .
\end{array}\right.
$$

Let $\mathcal{P}$ denote the set of probability measures on $\mathbb{R}$ with finite second-moment and endowed with the 1-Wasserstein distance.

Proposition 4. Consider the setting of Problem 1 with $\epsilon>0$ and suppose that Assumptions 1-2 hold. Then, (13) has a solution $m \in C([0, T], \mathcal{P})$. Moreover,

$$
d_{1}(m(t), m(t+h)) \leq C h^{1 / 2} .
$$

In addition, if Assumptions 3 and 4 hold, for any sequence $\varpi_{n} \rightarrow \varpi$ uniformly on $[0, T]$ and corresponing solutions $u_{n}$ of (12) and $m_{n}$ of (13), we have $m_{n} \rightarrow m$ in $C([0, T], \mathcal{P})$. 
Proof. The existence of a solution in $C([0, T], \mathcal{P})$ and the estimate in (14) were proven in [9]. We note that, for $\epsilon \leq \epsilon_{0}$, the constant $C$ can be chosen to depend only on $\epsilon_{0}$, on the problem data, and on $\|\varpi\|_{L^{\infty}}$. Thus, by the Ascoli-Arzela theorem, we have that $m_{n} \rightarrow m$ in $C([0, T], \mathcal{P})$ for some $m \in C([0, T], \mathcal{P})$. Because $\epsilon>0, m_{n} \rightarrow m$ in, for example, $L^{2}(\mathbb{R} \times[0, T])$. Moreover, (13) has a unique solution. Thus, it suffices to check that $m$ solves (13). Because $u_{x}^{n} \rightarrow u_{x}$, almost everywhere, by semiconcavity, we have for any $\psi \in C_{c}^{\infty}(\mathbb{R} \times[0, T])$

$$
\int_{0}^{T} \int_{\mathbb{R}} \psi_{x} D_{p} H\left(x, \varpi^{n}+u_{x}^{n}\right) m^{n} d x d t \rightarrow \int_{0}^{T} \int_{\mathbb{R}} \psi_{x} D_{p} H\left(x, \varpi+u_{x}\right) m d x d t,
$$

which gives that $m$ is a weak solution of (13).

Remark 6. Because $\epsilon>0, m$ enjoys further regularity by standard parabolic regularity. For example, since $D_{p} H\left(x, \varpi+u_{x}\right)$ is bounded, we have the energy estimate

$$
\frac{d}{d t} \int_{\mathbb{R}} m^{2} d x \leq C \int_{\mathbb{R}} m^{2} d x .
$$

which gives $m \in L^{\infty}\left([0, T], L^{2}(\mathbb{R})\right)$. In particular, $m$ is absolutely continuous. We note, that this estimate is not uniform in $\epsilon$ and hence cannot be used to derive the same result when $\epsilon=0$.

Next, we prove an estimate for solutions of the system comprising (12) and (13). This estimate is similar to the one in [15].

Proposition 5. Consider the setting of Problem 1 with $\epsilon>0$ and suppose that Assumptions 1 and 4 hold. Let $(u, m)$ solve 12 and 13. Then, there exists a constant $C$ that depends only on the problem data but not on $\epsilon$ such that

$$
\int_{0}^{T} \int_{\mathbb{R}} D_{p p}^{2} H u_{x x}^{2} m d x d t \leq C .
$$

Proof. We begin by differentiating (12) twice with respect to $x$, multiply by $m$, and integrate by parts using (13).

Remark 7. Formally, the previous estimates hold for $\epsilon=0$. However, the above proof requires that $u$ is three times differentiable, which is not usually the case. Nevertheless, the estimate in (15) is uniform in $\epsilon$ as can be seen from the proof.

Finally, we consider the price-supply relation. Due to Remark 4 and to the Lipschitz continuity of $u$ given by Proposition 1, there exists a unique $\vartheta_{0}$ such that

$$
\int_{\mathbb{R}} D_{p} H\left(x, \vartheta_{0}+u_{x}(x, 0)\right) \bar{m} d x=-\mathbf{Q}(0) .
$$

Moreover, $\vartheta_{0}$ is bounded by a constant that depends only on the problem data.

Next, we differentiate

$$
\int_{\mathbb{R}} D_{p} H\left(x, \varpi+u_{x}\right) m d x=-\mathbf{Q}(t)
$$

in time to get the identity

$$
\dot{\varpi} \int_{\mathbb{R}} D_{p p}^{2} H m d x+\int_{\mathbb{R}}\left[D_{p p}^{2} H u_{x t} m+D_{p} H m_{t}\right] d x=-\dot{\mathbf{Q}} .
$$


Differentiating (12) in $x$ and substituting (13) both quantities on the second term of the left-hand side of (17), we get the following identity

$$
\begin{aligned}
\int_{\mathbb{R}} D_{p p}^{2} H u_{x t} m+D_{p} H m_{t} d x= & \int_{\mathbb{R}} D_{p p}^{2} H\left(-\epsilon \Delta u_{x}+D_{p} H u_{x x}+D_{x} H\right) m d x \\
& +\int_{\mathbb{R}} D_{p} H\left(\epsilon \Delta m+\left(m D_{p} H\right)_{x}\right) d x .
\end{aligned}
$$

If Assumption 1 holds, we have by Remark 3 that $D_{x p}^{2} H=0$. Hence,

$$
\int_{\mathbb{R}} D_{p p}^{2} H u_{x t} m+D_{p} H m_{t} d x=\int_{\mathbb{R}} D_{p p}^{2} H D_{x} H m+\epsilon D_{p p p}^{3} H u_{x x}^{2} m d x .
$$

Accordingly, we have the identity

$$
\dot{\varpi} \int_{\mathbb{R}} D_{p p}^{2} H m=-\dot{\mathbf{Q}}-\int_{\mathbb{R}}\left(D_{p p}^{2} H D_{x} H+\epsilon D_{p p p}^{3} H u_{x x}^{2}\right) m .
$$

Thus, given $\varpi$, we solve (12) and (13) and define the following ordinary differential equation

$$
\left\{\begin{array}{l}
\dot{\vartheta}=\frac{-\dot{\mathbf{Q}}-\int_{\mathbb{R}} D_{p p}^{2} H\left(x, \varpi+u_{x}\right) D_{x} H\left(x, \varpi+u_{x}\right) m+\epsilon D_{p p p}^{3} H\left(x, \varpi+u_{x}\right) u_{x x}^{2} m d x}{\int_{\mathbb{R}} D_{p p}^{2} H\left(x, \varpi+u_{x}\right) m d x} \\
\vartheta(0)=\vartheta_{0},
\end{array}\right.
$$

where $\vartheta_{0}$ is determined by (16). Then, $(u, m, \varpi)$ solves (1) if $\varpi$ solves (20).

Proposition 6. Consider the setting of Problem 1 with $\epsilon>0$ and suppose that Assumptions 1-5 hold. Suppose that $\varpi^{n} \rightarrow \varpi$ uniformly in $C([0, T])$. Let $u^{n}$, $m^{n}$, and $\vartheta^{n}$ be the solutions to (12), (13), and 20 with $\varpi$ replaced by $\varpi^{n}$. Then, $\vartheta^{n}$ converges to $\vartheta$, uniformly in $C([0, T])$, where $\vartheta$ solves (20). Moreover, there exists a constant $C$ that depends only on the problem data but not on $\varpi$ such that $\|\vartheta\|_{W^{1,1}([0, T])} \leq C$.

Proof. The bound in $W^{1,1}([0, T])$ for $\vartheta$ results from the following observations. First, Remark 4 gives a uniform bound in $\varpi_{0}$. Second, $D_{p p}^{2} H\left(x, \varpi+u_{x}\right)$ is uniformly bounded by below by a positive constant by Assumption 5. Therefore, the denominator in the first equation in (20) is bounded by below. Since $\mathbf{Q}$ is smooth, $\mathbf{Q}$ is bounded. The bounds in Remark 5 and Assumption 5 bound the first term in the integral. Finally, the bound for $D_{p^{3}}^{3} H$ in Assumption 5 combined with the estimate in Proposition 5 imply that the last term under the integral is also bounded.

According to Proposition 3, the uniform convergence of $\varpi_{n} \rightarrow \varpi$ gives the convergence of $u_{x}^{n} \rightarrow u_{x}$, almost everywhere. In addition, Proposition 4 gives the convergence $m_{n} \rightarrow m$ in $C([0, T], \mathcal{P})$. Because $D_{p p}^{2} H$ is bounded from below by Assumption 5, we have the convergence of the right-hand side of (20) as follows, for any $\psi \in C([0, T])$,

$$
\int_{0}^{T} \psi \dot{\vartheta}_{n} d s \rightarrow \int_{0}^{T} \psi \dot{\vartheta} d s
$$

Also, because the family $\vartheta_{n}$ is equicontinuous, any subsequence has a further convergent subsequence that must converge to $\vartheta$. Thus, $\vartheta^{n} \rightarrow \vartheta$, uniformly.

With the preceding estimates, we can now prove a fixed-point result and show the existence of a solution for $\epsilon>0$. 
Proof of Theorem 1 - part 1, existence for $\epsilon \geq 0$. We begin by addressing the case $\epsilon>0$. According to Proposition 6, the map $\varpi \rightarrow \vartheta$ determined by (12), (13), and (20) is continuous in $C([0, T])$, bounded, and compact due to the $W^{1,1}$ bound for $\varpi$. Thus, by Schauder's fixed-point theorem, it has a fixed point.

Now, we examine the case $\epsilon=0$. The key difficulty is the continuity of the map $\varpi \rightarrow m$ in the case $\epsilon=0$. To overcome this difficulty, we use the vanishing viscosity method and the techniques in [15].

Let $\left(u^{\epsilon}, m^{\epsilon}, \varpi^{\epsilon}\right)$ solve (1) with $\epsilon>0$. By the above, we have that $\varpi^{\epsilon}$ is uniformly bounded. Moreover, by Proposition $1, u^{\epsilon}$ is uniformly locally bounded and equiLipschitz. Therefore, as $\epsilon \rightarrow 0$, extracting a subsequence if necessary, by AscoliArzela theorem, $\varpi^{\epsilon} \rightarrow \varpi$ and $u^{\epsilon} \rightarrow u$ where $u$ is a viscosity solution of (12).

Now, we introduce a phase-space measure $\mu^{\epsilon}$ as follows

$$
\int_{0}^{T} \int_{\mathbb{R}^{2}} \psi(x, p, t) d \mu^{\epsilon}(x, p, t)=\int_{0}^{T} \int_{\mathbb{R}} \psi\left(x, \varpi^{\epsilon}+u_{x}^{\epsilon}, t\right) m^{\epsilon} d x d t
$$

for all $\psi \in C_{b}(\mathbb{R} \times \mathbb{R} \times[0, T])$. This construction is inspired by a similar one in weak KAM theory, see, for example, $[7,8,15,16,17]$. There, these measures encode the Hamiltonain flow of certain probability measures on phase space (positionmomentum coordinates) through the corresponing Hamiltonian dynamics. Because $m^{\epsilon} \in C([0, T], \mathcal{P})$ with a modulus of continuity that is uniform in $\epsilon$ and $\varpi^{\epsilon}+u_{x}^{\epsilon}$ is uniformly bounded, the sequence $\mu^{\epsilon}$ is tight. Therefore, as $\epsilon \rightarrow 0$, we have $\mu^{\epsilon} \rightarrow \mu$; that is

$$
\int_{0}^{T} \int_{\mathbb{R}^{2}} \psi d \mu^{\epsilon} \rightarrow \int_{0}^{T} \int_{\mathbb{R}^{2}} \psi d \mu
$$

Moreover, due to the strict convexity of the Hamiltonian, arguing as in [15], we have for any $\varphi(x, t): \mathbb{R} \times[0, T] \rightarrow \mathbb{R}$, of class $C^{1}$ that

$$
\begin{aligned}
\int_{0}^{T} & \int_{\mathbb{R}^{2}} \varphi_{t}(x, t)-D_{p} H(x, p) D_{x} \varphi(x, t) d \mu \\
\quad & \int_{\mathbb{R}} \varphi(x, T) m(x, T) d x-\int_{\mathbb{R}} \varphi(x, 0) \bar{m}(x) d x .
\end{aligned}
$$

Next, we fix $\delta>0$ and consider a standard mollifier, $\eta_{\delta}$; that is, a non-negative $C^{\infty}$ function, radial, compactly supported on $B_{\delta}(0)$, with $\int_{\mathbb{R}} \eta_{\delta}(y) d y=1$. We define

$$
v^{\delta}(x)=\left(\eta_{\delta} * u\right)(x)=\int_{\mathbb{R}} \eta_{\delta}(x-y) u(y) d y .
$$

We note that $\left|D^{2} v^{\delta}\right| \leq \frac{C}{\delta^{2}}$. Then, using the uniform convexity of the Hamiltonian, we get

$$
-v_{t}^{\delta}+\eta_{\delta} *\left|u_{x}-v_{x}^{\delta}\right|^{2}+H\left(x, \varpi+v_{x}^{\delta}\right) \leq O(\delta) .
$$

Therefore, $w=v^{\delta}-u^{\epsilon}$ satisfies

$$
\begin{aligned}
& -w_{t}+D_{p} H\left(x, \varpi^{\epsilon}+u_{x}^{\epsilon}\right) w_{x}-\epsilon w_{x x} \\
& +\eta_{\delta} *\left|u_{x}-v_{x}^{\delta}\right|^{2}+\gamma\left|\varpi+v_{x}^{\delta}-\varpi^{\epsilon}-u_{x}^{\epsilon}\right|^{2} \leq O(\delta)+O\left(\frac{\epsilon}{\delta^{2}}\right) .
\end{aligned}
$$

Integrating with respect to $m^{\epsilon}$, we conclude that

$$
\int_{0}^{T} \int_{\mathbb{R}^{2}} \eta_{\delta} *\left|u_{x}-v_{x}^{\delta}\right|^{2}+\gamma\left|\varpi+v_{x}^{\delta}-p\right|^{2} d \mu^{\epsilon} \leq O(\delta)+O\left(\frac{\epsilon}{\delta^{2}}\right)+\left\|v^{\delta}-u^{\epsilon}\right\|_{L^{\infty}} .
$$


Next, we let $\epsilon \rightarrow 0$, to get

$$
\int_{0}^{T} \int_{\mathbb{R}^{2}} \eta_{\delta} *\left|u_{x}-v_{x}^{\delta}\right|^{2}+\gamma\left|\varpi+v_{x}^{\delta}-p\right|^{2} d \mu \leq O(\delta) .
$$

Finally, by letting $\delta \rightarrow 0$, we conclude that $m$-almost every point is a point of approximate continuity of $u_{x}$. Therefore, $v_{x}^{\delta} \rightarrow u_{x}$ almost everywhere. Hence, $p=\varpi+u_{x} \mu$-almost everywhere. Therefore, we obtain

$$
\begin{aligned}
\int_{0}^{T} & \int_{\mathbb{R}^{2}}\left(\psi_{t}-D_{p} H\left(x, \varpi+u_{x}\right) D_{x} \psi\right) d \mu \\
= & \int_{0}^{T} \int_{\mathbb{R}}\left(\psi_{t}-D_{p} H\left(x, \varpi+u_{x}\right) D_{x} \psi\right) m d x d t \\
& =\int_{\mathbb{R}} \psi(x, T) m(x, T) d x-\int_{\mathbb{R}} \psi(x, 0) \bar{m}(x) d x,
\end{aligned}
$$

which gives that $m$ solves (13) with $\epsilon=0$.

Note also, that the preceding reasoning implies that $u$ is differentiable almost everywhere with respect to $m$.

Finally, we record two additional results for (1). The first is an energy estimate that is similar to the energy estimates in mean-field games - also called first-order estimates in Section 6.2 of [23].

Proposition 7. Let $(u, m, \varpi)$ be the solution of Problem 1 constructed in Theorem 1. Suppose that there exists $C>0$ such that

$$
p D_{p} H(x, p)-H(x, p) \geq \frac{1}{C} H(x, p)-C .
$$

Then,

$$
\int_{0}^{T} \int_{\mathbb{R}} H\left(x, \varpi+u_{x}\right)\left(m_{0}+m\right) d x d t \leq C .
$$

Proof. We take the first equation in (1) and multiply it by $\bar{m}-m$, and the second equation by $u-\bar{u}$. Adding the resulting expressions and integrating by parts results in the desired estimate.

The last result in this section concerns the regularity of the solutions (12) in the case where both the potential and terminal data are convex.

Proposition 8. Suppose that $\epsilon=0$, that Assumptions 1, 3, and 6 hold and let $\varpi$ be a Lipschitz function. Then, the solution to (12) is differentiable in $x$ for every $x \in \mathbb{R}$. Moreover, $u_{x x}$ is bounded.

Proof. Due to Assumption 6, we see that $u(x, t)$ is convex in $x$ by direct inspection of the variational problem (6). By Proposition 2, $u$ is semiconcave in $x$. This gives the bound for $u_{x x}$ and the differentiability of $u$ in $x$.

The preceding proposition implies the regularity of the solutions of Problem 1, as stated in the next Corollary.

Corollary 1. Suppose that Assumptions 1-6 hold and that $\epsilon=0$. Then, there exists a solution $(u, m, \varpi)$ of Problem 1 with $u$ differentiable in $x$ for every $x$ and $u_{x x}$ bounded. Moreover, $m$ is also bounded. 
Proof. The result follows by combining Proposition 8 which gives the existence of a solution to (12) with $u_{x x}$ uniformly bounded. Because of this, the transport equation has drift $\varpi+u_{x}$ which has locally Lipschitz coefficients. Hence, by the method of characteristics, it has a unique weak solution in $L^{\infty}$. Therefore, in this case, we do not need to use the parabolic regularization $(\epsilon>0)$ to use a fixed-point theorem to establish a solution as this can be done directly for $\epsilon=0$.

Proof of Theorem 1 - part 2, additional regularity for $\epsilon=0$. Additional regularity for the case where Assumption 6 holds and $\epsilon=0$ follows from Corollary 1.

\section{UNIQUENESS}

Now, we examine the uniqueness of solutions. We begin by observing that (1) can be written as a monotone operator. As a consequence, we obtain a uniqueness result. While in this paper we only use the monotonicity to establish uniqueness, this observation is an interesting fact that can be used, for example, to construct numerical methods in a similar manner to what was done in [1] or [25].

We set

$$
\Omega_{T}=\mathbb{R} \times[0, T],
$$

and

$$
\begin{aligned}
D & =\left(C^{\infty}\left(\Omega_{T}\right) \cap C([0, T], \mathcal{P})\right) \times\left(C^{\infty}\left(\Omega_{T}\right) \cap W^{1, \infty}\left(\Omega_{T}\right)\right) \times C^{\infty}([0, T]), \\
D_{+} & =\{(m, u, \varpi) \in D \text { s.t. } m>0\}, \\
D^{b} & =\{(m, u, \varpi) \in D \text { s.t. } m(x, 0)=\bar{m}(x), u(x, T)=\bar{u}(x)\}, \\
D_{+}^{b} & =D^{b} \cap D_{+},
\end{aligned}
$$

Then, we define $A: D_{+}^{b} \rightarrow D$ as

$$
\begin{aligned}
A\left[\begin{array}{c}
m \\
u \\
\varpi
\end{array}\right] & =A_{1}\left[\begin{array}{c}
m \\
u \\
\varpi
\end{array}\right]+A_{2}\left[\begin{array}{c}
m \\
u \\
\varpi
\end{array}\right] \\
& =\left[\begin{array}{c}
u_{t}+\epsilon u_{x x} \\
m_{t}-\epsilon m_{x x} \\
0
\end{array}\right]+\left[\begin{array}{c}
-\operatorname{div}\left(m D_{p} H\left(x, \varpi+u_{x}\right)\right) \\
\int_{\Omega} m D_{p} H\left(x, \varpi+u_{x}\right) d x+\mathbf{Q}(t)
\end{array}\right] .
\end{aligned}
$$

Furthermore, for $w=(m, u, \varpi), \tilde{w}=(\tilde{m}, \tilde{u}, \tilde{\varpi}) \in D$, we set

$$
\langle w, \tilde{w}\rangle=\int_{\Omega_{T}}(m \tilde{m}+u \tilde{u}) d x d t+\int_{0}^{T} \varpi \tilde{\varpi} d t .
$$

Then, $A$ is a monotone operator if

$$
\langle A[w]-A[\tilde{w}], w-\tilde{w}\rangle \geq 0 \quad \text { for all } \quad w, \tilde{w} \in D_{+}^{b} .
$$

Under the convexity of the map $p \mapsto H(x, p), A$ is a monotone operator.

Proposition 9. Suppose the map $p \mapsto H(x, p)$ is convex. Then $A$ is a monotone operator. 
Proof. Let $w=(m, u, \varpi), \tilde{w}=(\tilde{m}, \tilde{u}, \tilde{\varpi}) \in D_{+}^{b}$. Then, integrating by parts, we obtain

$$
\begin{aligned}
& \left\langle A_{1}[w]-A_{1}[\tilde{w}], w-\tilde{w}\right\rangle \\
= & \int_{\Omega_{T}}\left((u-\tilde{u})_{t}+\epsilon \Delta(u-\tilde{u})\right)(m-\tilde{m})+\int_{\Omega_{T}}\left((m-\tilde{m})_{t}-\epsilon \Delta(m-\tilde{m})\right)(u-\tilde{u}) \\
= & 0,
\end{aligned}
$$

because $u-\tilde{u}$ and $m-\tilde{m}$ vanish at $t=0$ and $t=T$. Furthermore, we have that

$$
\begin{aligned}
& \left\langle A_{2}[w]-A_{2}[\tilde{w}], w-\tilde{w}\right\rangle \\
= & -\int_{\Omega_{T}}\left(H\left(x, u_{x}+\varpi\right)-H\left(x, \tilde{u}_{x}+\tilde{\varpi}\right)\right)(m-\tilde{m}) d x d t \\
& -\int_{\Omega_{T}} \operatorname{div}\left(m D_{p} H\left(x, u_{x}+\varpi\right)-\tilde{m} D_{p} H\left(x, \tilde{u}_{x}+\tilde{\varpi}\right)\right)(u-\tilde{u}) d x d t \\
& +\int_{0}^{T}(\varpi-\tilde{\varpi}) \int_{\mathbb{R}}\left(m D_{p} H\left(x, u_{x}+\varpi\right)-\tilde{m} D_{p} H\left(x, \tilde{u}_{x}+\tilde{\varpi}\right)\right) d x d t \\
= & -\int_{\Omega_{T}}\left(H\left(x, u_{x}+\varpi\right)-H\left(x, \tilde{u}_{x}+\tilde{\varpi}\right)\right)(m-\tilde{m}) d x d t \\
& +\int_{\Omega_{T}}\left(m D_{p} H\left(x, u_{x}+\varpi\right)-\tilde{m} D_{p} H\left(x, \tilde{u}_{x}+\tilde{\varpi}\right)\right)\left(u_{x}-\tilde{u}_{x}\right) d x d t \\
& +\int_{\Omega_{T}}\left(m D_{p} H\left(x, u_{x}+\varpi\right)-\tilde{m} D_{p} H\left(x, \tilde{u}_{x}+\tilde{\varpi}\right)\right)(\varpi-\tilde{\varpi}) d x d t \\
= & \int_{\Omega_{T}} m\left(H\left(x, \tilde{u}_{x}+\tilde{\varpi}\right)-H\left(x, u_{x}+\varpi\right)-\left(\tilde{u}_{x}+\tilde{\varpi}-u_{x}-\varpi\right) D_{p} H\left(x, u_{x}+\varpi\right)\right) d x d t \\
& +\int_{\Omega_{T}} \tilde{m}\left(H\left(x, u_{x}+\varpi\right)-H\left(x, \tilde{u}_{x}+\tilde{\varpi}\right)-\left(u_{x}+\varpi-\tilde{u}_{x}-\tilde{\varpi}\right) D_{p} H\left(x, \tilde{u}_{x}+\tilde{\varpi}\right)\right) d x d t \\
\geq &
\end{aligned}
$$

$\geq 0$,

by the convexity of $p \mapsto H(x, p)$. Combining the previous inequalities, we conclude that

$$
\begin{aligned}
& \langle A[w]-A[\tilde{w}], w-\tilde{w}\rangle \\
= & \left\langle A_{1}[w]-A_{1}[\tilde{w}], w-\tilde{w}\right\rangle+\left\langle A_{2}[w]-A_{2}[\tilde{w}], w-\tilde{w}\right\rangle \geq 0
\end{aligned}
$$

Now, we discuss the last part of the proof of Theorem 1.

Proof of Theorem 1 - part 3, uniqueness. Let $(m, u, \varpi)$ and $(\tilde{m}, \tilde{u}, \tilde{\varpi})$ solve Problem 1. If $\epsilon>0$ or if $\epsilon=0$ and Assumption 6 holds, we have $m$ and $\tilde{m}$ are absolutely continuous with respect to the Lebesgue measure. Thus, the computations in the proof of Proposition 9, combined with the uniform convexity of $H$ in Assumption 
5 , give

$$
\int_{0}^{T} \int_{\mathbb{R}}\left|\varpi+u_{x}-\tilde{\varpi}-\tilde{u}_{x}\right|^{2}(\tilde{m}+m)=0 .
$$

Therefore, $\varpi+u_{x}=\tilde{\varpi}+\tilde{u}_{x}$ almost everywhere. In both cases, this implies

$$
u_{t}=\tilde{u}_{t},
$$

almost everywhere and, thus, $u=\tilde{u}$. Finally, the uniqueness of the Fokker-Planck equation, for $\epsilon>0$ or for the transport equation, when $\epsilon=0$ and Assumption 6 holds, give $m=\tilde{m}$.

\section{LiNEAR-QUADRATIC MODELS}

Here, we consider linear-quadratic price models. First, we examine the case without a potential and determine an explicit solution. Then, we introduce a quadratic potential that accounts for charge level preferences. In this last case, we describe a procedure to solve the problem, up to the inversion of Laplace transforms and solution of ordinary differential equations.

6.1. State-independent quadratic cost. First, we consider the quadratic stateindependent cost

$$
\ell(t, \alpha)=\frac{c}{2} \alpha^{2}+\alpha \varpi(t),
$$

where $c$ is a constant that accounts for the usage-depreciation of the battery. The corresponding MFG is

$$
\left\{\begin{array}{l}
-u_{t}+\frac{\left(\varpi(t)+u_{x}\right)^{2}}{2 c}=0 \\
m_{t}-\frac{1}{c}\left(m\left(\varpi(t)+u_{x}\right)\right)_{x}=0 \\
\frac{1}{c} \int_{\mathbb{R}}\left(\varpi(t)+u_{x}\right) m d x=-\mathbf{Q}(t) .
\end{array}\right.
$$

The stored energy by each agent follows optimal trajectories that solve the Euler Lagrange equation:

$$
c \ddot{\mathbf{x}}+\dot{\varpi}=0 .
$$

Integrating the previous equation in time, we get

$$
\dot{\mathbf{x}}(t)=\frac{1}{c}(\theta-\varpi(t)),
$$

where $\theta$ is time independent. Next, by differentiating the Hamilton-Jacobi equation, we get

$$
-\left(u_{x}\right)_{t}+\left(u_{x}+\varpi\right) \frac{u_{x x}}{c}=0 .
$$

Using the previous equation, taking into account the transport equation, and integrating by parts, we have

$$
\begin{aligned}
& \frac{d}{d t} \int_{\mathbb{R}} u_{x} m d x=\int_{\mathbb{R}} u_{x t} m+u_{x} m_{t}=\int_{\mathbb{R}} u_{x t} m+\frac{1}{c} u_{x}\left(m\left(\varpi+u_{x}\right)\right)_{x} \\
& =\frac{1}{c} \int_{\mathbb{R}}\left(\varpi+u_{x}\right) u_{x x} m-u_{x x} m\left(\varpi+u_{x}\right) d x=0,
\end{aligned}
$$

assuming that $m$ has fast enough decay at infinity.

Thus, the supply vs demand balance condition becomes

$$
\mathbf{Q}(t)=-\frac{1}{c} \int_{\mathbb{R}}\left(u_{x}+\varpi\right) m d x=\frac{1}{c}(\Theta-\varpi),
$$


where

$$
\Theta=-\int_{\mathbb{R}} u_{x} m d x
$$

is constant. From the above, we obtain the following linear price-supply relation

$$
\varpi=\Theta-c \mathbf{Q}(t)
$$

Integrating (24) in time and taking into account the linear price-supply relation (26), we gather

$$
\mathbf{x}(T)=\mathbf{x}(t)+\frac{1}{c} \int_{t}^{T}(\theta-\varpi(s)) d s=x+\frac{T-t}{c}(\theta-\Theta)+\int_{t}^{T} \mathbf{Q}(s) d s .
$$

Accordingly, $u$ is given by the optimization problem

$$
\begin{aligned}
u(x, t)= & \inf _{\theta} \int_{t}^{T}\left[\frac{(\theta-\Theta+c \mathbf{Q}(s))^{2}}{2 c}+\frac{1}{c}(\theta-\Theta+c \mathbf{Q}(s))(\Theta-c \mathbf{Q}(s))\right] d s \\
& +\bar{u}\left(x+\frac{(\theta-\Theta)}{c}(T-t)+K(t)\right),
\end{aligned}
$$

where

$$
K(t)=\int_{t}^{T} Q(s) d s
$$

By setting $\mu=\theta-\Theta$, we get

$$
\begin{aligned}
u(x, t)= & \inf _{\mu} \int_{t}^{T}\left[\frac{(\mu+c \mathbf{Q}(s))^{2}}{2 c}+\frac{1}{c}(\mu+c \mathbf{Q}(s))(\Theta-c \mathbf{Q}(s))\right] d s \\
& +\bar{u}\left(x+\frac{\mu}{c}(T-t)+K(t)\right) .
\end{aligned}
$$

Thus, given $\Theta$, we determine a function, $u^{\Theta}$, solving the preceding minimization problem. For that, we expand the integral to get

$$
\begin{array}{r}
u^{\Theta}(x, t)=\inf _{\mu}\left[\frac{T-t}{2 c} \mu^{2}+\frac{1}{c}(T-t) \Theta \mu\right. \\
+\int_{t}^{T}\left(\Theta-c \frac{\mathbf{Q}(s)}{2}\right) \mathbf{Q}(s) d s \\
\left.+\bar{u}\left(x+\frac{\mu}{c}(T-t)+K(t)\right)\right] .
\end{array}
$$

Next, we take the derivative of the right-hand side of the prior identity with respect to $\mu$ and obtain the relation

$$
\mu+\bar{u}_{x}(\mathbf{x}(T))=-\Theta .
$$

If $\bar{u}$ is a convex function, the preceding equation has a unique solution, $\mu(\Theta)$ for each given $\Theta$. Thus, given $\Theta$, we obtain a solution, $u^{\Theta}$ for the Hamilton-Jacobi equation. Finally, we use the resulting expression for $u^{\Theta}$ in (25) at $t=0$ to obtain the following condition for $\Theta$ :

$$
\Theta=-\int_{\mathbb{R}} u_{x}^{\Theta}(x, 0) m_{0}(x) d x .
$$

Solving the preceding equation, we obtain $\Theta$ and hence $\varpi$ using the price-supply relation, (26).

As an example, we consider the terminal cost

$$
\bar{u}(y)=\frac{\gamma}{2}(y-\zeta)^{2}
$$


Solving(28), we obtain

$$
\mu=-\frac{\gamma(K(t)+x-\zeta)+\Theta}{1+\gamma \frac{T-t}{c}} .
$$

Accordingly, we have

$$
\begin{aligned}
& u^{\Theta}(x, t)= \\
& \frac{\gamma(K(t)+x-\zeta)^{2}+\frac{(t-T)}{c} \Theta(2 \gamma(K(t)+x-\zeta)+\Theta)}{2\left(1+\gamma \frac{T-t}{c}\right)} \\
& +\Theta K(t)-c \int_{t}^{T} \frac{\mathbf{Q}^{2}(s)}{2} d s .
\end{aligned}
$$

Therefore,

$$
u_{x}(x, t)=\gamma \frac{K(t)+x-\zeta-\frac{(T-t)}{c} \Theta}{1+\gamma \frac{T-t}{c}}
$$

Using the previous expression for $t=0$ in (29), we obtain the following equation for $\Theta$

$$
\Theta=-\gamma \frac{K(0)+\bar{x}-\zeta-\frac{T}{c} \Theta}{1+\gamma \frac{T}{c}}
$$

where

$$
\bar{x}=\int_{\mathbb{R}} x m_{0} d x .
$$

Thus,

$$
\Theta=-\gamma(K(0)+\bar{x}-\zeta)
$$

Therefore, using (26), we obtain

$$
\varpi=-\gamma(K(0)+\bar{x}-\zeta)-c \mathbf{Q} .
$$

Finally, we use the above results and conclude that each agent dynamics is

$$
\left\{\begin{array}{l}
\dot{\mathbf{x}}=\frac{(\bar{x}-x) \gamma}{1+\frac{T}{c} \gamma}+\mathbf{Q} \\
\mathbf{x}(0)=x
\end{array}\right.
$$

In alternative, using

$$
\dot{\mathbf{x}}(t)=-\frac{\varpi+u_{x}(\mathbf{x}(t), t)}{c}
$$

we have

$$
\left\{\begin{array}{l}
\dot{\mathbf{x}}(t)=\frac{(\overline{\mathbf{x}}(t)-\mathbf{x}(t)) \gamma}{1+\frac{T-t}{c} \gamma}+\mathbf{Q} \\
\mathbf{x}(0)=x
\end{array}\right.
$$

where

$$
\overline{\mathbf{x}}(t)=\int_{\mathbb{R}} x m(x, t) d x .
$$

Averaging (32) with respect to $m$, we obtain

$$
\dot{\overline{\mathbf{x}}}(t)=\mathbf{Q}(t),
$$


which is simply the conservation of energy. Thus, the trajectory of an individual agent can be computed by combining (32) with (33) into the system

$$
\left\{\begin{array}{l}
\dot{\mathbf{x}}(t)=\frac{(\overline{\mathbf{x}}(t)-\mathbf{x}(t)) \gamma}{1+\frac{T-t}{c} \gamma}+\mathbf{Q}(t) \\
\dot{\overline{\mathbf{x}}}(t)=\mathbf{Q}
\end{array}\right.
$$

The previous system is a closed system of ordinary differential equations that only involves $\mathbf{Q}$ and the parameters of the problem. Surprisingly, it also does not depend on $\zeta$. This is due to the fact that the average of the position of the agents is determined by $\mathbf{Q}$. Hence, the only way agents can improve their value function is by getting close to each other. This is seen in the mean-reverting structure in (32).

6.2. Quadratic cost with potential. Now, we consider a running cost with a quadratic potential. This potential penalizes the agents when the charge or stored energy deviates too much from a set point, $\kappa$. This penalty has the form of $\frac{\eta}{2}(x-\kappa)^{2}$, where $\eta$ measures the strength of the penalty. Thus, for $c=1$, we have

$$
\ell(t, x, \alpha)=\frac{\alpha^{2}}{2}+\alpha \varpi(t)+\frac{\eta}{2}(x-\kappa)^{2} .
$$

The corresponding MFG is

$$
\left\{\begin{array}{l}
-u_{t}+\frac{\left(\varpi(t)+u_{x}\right)^{2}}{2}-\frac{\eta}{2}(x-\kappa)^{2}=0 \\
m_{t}-\left(m\left(\varpi(t)+u_{x}\right)\right)_{x}=0 \\
\int\left(\varpi(t)+u_{x}\right) m=-\mathbf{Q}(t) .
\end{array}\right.
$$

Differentiating the Hamilton-Jacobi equation, we conclude that

$$
-\left(u_{x}\right)_{t}+\left(u_{x}+\varpi\right) u_{x x}-\eta(x-\kappa)=0 .
$$

We define the following quantities

$$
\Pi=\int_{\mathbb{R}} u_{x} m \text { and } \Xi=\int_{\mathbb{R}} x m .
$$

Taking the time derivative on the first quantity and using the transport equation, we get

$$
\begin{aligned}
\dot{\Pi} & =\int_{\mathbb{R}} u_{x t} m+u_{x} m_{t} \\
& =\int_{\mathbb{R}}\left(\varpi+u_{x}\right) u_{x x} m-\eta \int_{\mathbb{R}}(x-\kappa) m+\int_{\mathbb{R}} u_{x}\left(m\left(\varpi+u_{x}\right)\right)_{x} \\
& =\int_{\mathbb{R}}\left(\varpi+u_{x}\right) u_{x x} m-\int_{\mathbb{R}} u_{x x} m\left(\varpi+u_{x}\right)-\eta \int_{\mathbb{R}}(x-\kappa) m .
\end{aligned}
$$

Simplifying the preceding expression, we obtain

$$
\dot{\Pi}=-\eta(\Xi-\kappa) .
$$


Next, we take the transport equation, multiply it by $x$, and integrate by parts, to get

$$
\begin{aligned}
\dot{\Xi} & =\frac{d}{d t} \int_{\mathbb{R}} x m=\int_{\mathbb{R}} x m_{t}=\int_{\mathbb{R}} x\left(m\left(\varpi+u_{x}\right)\right)_{x} \\
& =-\int_{\mathbb{R}} m\left(\varpi+u_{x}\right)+\left[x \left(\left.m\left(\varpi+u_{x}\right)\right|_{\Omega}\right.\right. \\
& =-\varpi-\int_{\mathbb{R}} u_{x} m .
\end{aligned}
$$

Thus, we conclude that

$$
\dot{\Xi}=-\varpi-\Pi \text {. }
$$

Therefore, we obtain the following averaged dynamics

$$
\left\{\begin{array}{l}
\dot{\Xi}=-\varpi-\Pi \\
\dot{\Pi}=-\eta(\Xi-\kappa)
\end{array}\right.
$$

Taking the time derivative of the second equation and using the first equation, we get

$$
\ddot{\Pi}-\eta \Pi=\eta(\varpi+\kappa) .
$$

The preceding equation has the following solution

$$
\Pi=-\kappa+e^{\sqrt{\eta} t} C_{1}+e^{-\sqrt{\eta} t} C_{2}+\frac{\sqrt{\eta}}{2} \int_{0}^{t}\left(e^{\sqrt{\eta}(t-s)}-e^{-\sqrt{\eta}(t-s)}\right) \varpi(s) d s .
$$

Moreover, at $t=0$, we have

$$
\dot{\Pi}(0)=-\eta(\Xi(0)-\kappa)=-\eta(\bar{x}-\kappa),
$$

where

$$
\bar{x}=\int_{\mathbb{R}} x \bar{m} .
$$

Thus, we need an additional constant to determine $\Pi(0)$. Given this constant, from the constraint equation in (34), we get

$$
\begin{aligned}
\varpi_{\Pi(0)}(t) & =-\Pi-\mathbf{Q}(t) \\
& =f_{\Pi(0)}(t)-\frac{\sqrt{\eta}}{2} \int_{0}^{t}\left(e^{\sqrt{\eta}(t-s)}-e^{-\sqrt{\eta}(t-s)}\right) \varpi_{\Pi(0)}(s) d s,
\end{aligned}
$$

where $f_{\Pi(0)}(t)=\kappa-e^{\sqrt{\eta} t} C_{1}-e^{-\sqrt{\eta} t} C_{2}-\mathbf{Q}(t)$, and $C_{1}$ and $C_{2}$ are determined by the value of $\dot{\Pi}(0)$ and by the unknown value $\Pi(0)$.

The preceding equation is a Volterra integral equation of the second kind with a separable kernel. In principle, we can solve this equation using Laplace's transform. The previous equation is of the form

$$
\varpi_{\Pi(0)}(t)=f_{\Pi(0)}(t)-\lambda\left(k * \varpi_{\Pi(0)}\right)(t),
$$

where

$$
k(t)=-\frac{\sqrt{\eta}}{2}\left(e^{\sqrt{\eta} t}-e^{-\sqrt{\eta} t}\right)
$$

and $(k * \varpi)=\int_{0}^{t} k(t-s) \varpi(s) d s$ denotes the convolution product of the kernel $k$ with $\varpi$. 
Let $\mathcal{L}$ denote the Laplace transform. Because $\mathcal{L}\{(k * \varpi)(t)\}=\mathcal{L}\{k(t)\} \mathcal{L}\{\varpi(t)\}$, applying the Laplace transform to (35) yields

$$
\mathcal{L}\{\varpi(t)\}=\mathcal{L}\left\{f_{\Pi(0)}(t)\right\}+\lambda \mathcal{L}\{k(t)\} \mathcal{L}\{\varpi(t)\} .
$$

Simplifying the above equation, we obtain

$$
\varpi_{\Pi(0)}(t)=\mathcal{L}^{-1}\left\{\frac{\mathcal{L}\left\{f_{\Pi(0)}(t)\right\}}{1-\lambda \mathcal{L}\{k(t)\}}\right\}
$$

where $\mathcal{L}^{-1}$ is the inverse Laplace transform.

Finally, we take the resulting expression for $\varpi_{\Pi(0)}$ into the Hamilton-Jacobi equation, solve it and obtain a function $u_{\Pi(0)}(x, t)$. Then, the value $\Pi_{0}$ is determined implicitly by the equation

$$
\Pi(0)=\int_{\mathbb{R}}\left(u_{\Pi(0)}\right)_{x}(x, 0) m_{0} d x .
$$

In the case of quadratic terminal data,

$$
u(x, T)=\frac{\gamma}{2}(x-\zeta)^{2},
$$

we can reduce the solution of the Hamilton-Jacobi equation into solving ordinary differential equations. For that, we look for a solution

$$
u(x, t)=\theta_{0}(t)+\theta_{1}(t) x+\theta_{2}(t) x^{2}
$$

satisfying

$$
u(x, T)=\frac{\gamma}{2}(x-\zeta)^{2}=\theta_{0}(T)+\theta_{1}(T) x+\theta_{2}(T) x^{2} .
$$

Then, the first equation in (34) becomes

$$
-\left(\dot{\theta}_{0}+\dot{\theta}_{1}(t) x+\dot{\theta}_{2}(t) x^{2}\right)+\frac{\left(\varpi(t)+\theta_{1}(t)+2 \theta_{2}(t) x\right)^{2}}{2 c}-\frac{\eta}{2}(x-\kappa)^{2}=0 .
$$

Thus, by matching powers of $x$, we obtain differential equations for $\theta_{i}, 0 \leq i \leq 2$. The resulting expression can be used in (36) to obtain the solution.

\section{Real Data}

In this section, we use real data of daily energy consumption in the UK, during a twenty-four hour period. The data is available online at https://www . nationalgrid.com/uk/. In Figure 1, we plot the power supply oscillation $\mathbf{Q}$ (which is simply the negative of the demand) normalized to have mean zero over 24 hours.

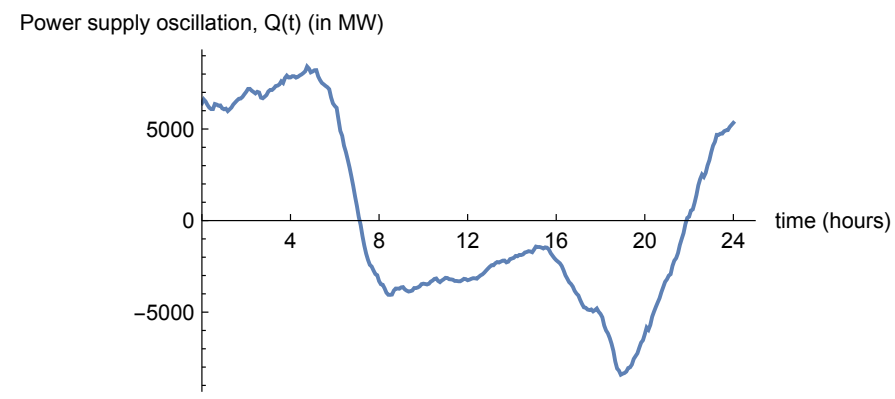

FIG. 1. Normalized electricity production $\mathbf{Q}$. 
We compare our price-formation model with the MFG model presented in [12]. In that model, the energy price is a function of the aggregate consumption. In that case, the price is not determined by a supply vs demand condition and, thus, there may be an energy imbalance. Here, we consider the state-independent quadratic cost model from Section 6.1. In our model, the price depends only on the constant that accounts for battery's wear and tear. This constant can be empirically estimated, but, here, we calibrate our model against the model in [12] using a least squares approach. Let $\vartheta$ be the priced computed in [12]. According to (26), the price given is $\varpi^{c, \Theta}=\Theta-c \mathbf{Q}$. Thus, we estimate the value of $c$, by solving the minimization problem

$$
\min _{c, \Theta \in \mathbb{R}}\left\|\varpi^{c, \Theta}-\vartheta\right\|_{2}^{2}=\min _{c, \Theta \in \mathbb{R}}\|\Theta-c \mathbf{Q}-\vartheta\|_{2}^{2},
$$

and, using $N=10^{6}$ agents, we obtained $c=0.00172 \$(\mathrm{~kW})^{-2} h^{-1}$.

The price given by our model is plotted in Figure 2. We predict smaller peak oscillations and thus, our methods may help stabilize the market.

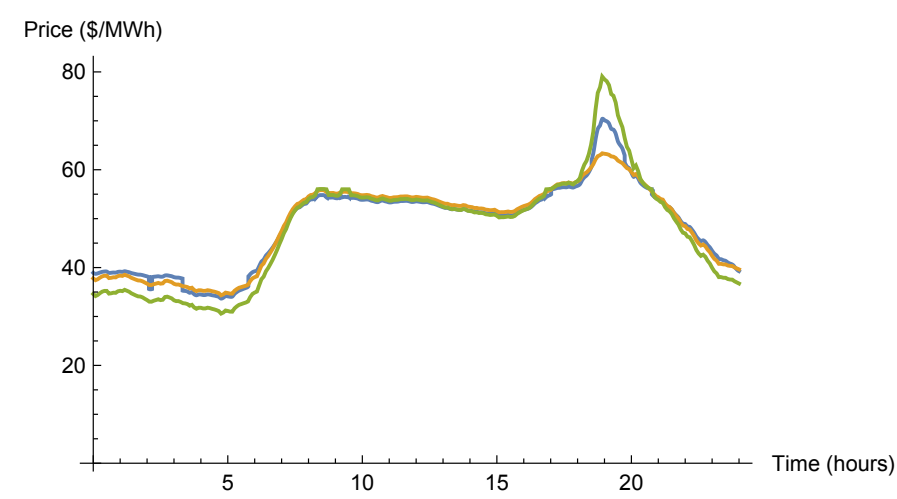

FIG. 2. Evolution of electricity price during a twenty-four hour period. In green, we plot the energy's price when no batteries are connected to the grid. In blue, we plot the price with batteries connected to the grid and the price is given by the model in [12]. In yellow, we plot the price corresponding to our model.

\section{Conclusions And EXtensions}

Here, we described a model for price formation in electricity markets, proved the well-posedness of the problem, and developed methods to compute the solutions. Our model has a minimal number of features and fits well real data. In addition, our model may have stabilizing properties of the price at peak consumption.

Several extensions of our model are of interest. First, we can consider the case where the supply $\mathbf{Q}(\varpi, t)$ depends on price. Provided the supply increases with the price, which is a natural assumption from the economic point of view, the solvability conditions are similar. In particular, (19) becomes

$$
\dot{\varpi}\left[\frac{\partial \mathbf{Q}}{\partial \varpi}+\int_{\mathbb{R}} D_{p p}^{2} H m d x\right]=-\frac{\partial \mathbf{Q}}{\partial t}-\int_{\mathbb{R}}\left[D_{p p}^{2} H D_{x} H m+\epsilon D_{p p p}^{3} H u_{x x}^{2} m\right] d x .
$$


Thus, we obtain similar bounds for $\dot{\varpi}$ if $\frac{\partial \mathbf{Q}}{\partial \varpi} \geq 0$. Therefore, the existence theory follows a similar argument. Moreover, if $\frac{\partial \mathbf{Q}}{\partial \varpi} \geq 0$, the operator $A$ in section (5) is monotone and, therefore, uniqueness of solution holds.

In real applications, $\mathbf{Q}$ may depend on delayed prices. While this does not fit directly into our framework, we can consider a Taylor expansion:

$$
\begin{aligned}
\mathbf{Q}(\varpi(t-\tau), t) \simeq \mathbf{Q}(\varpi(t), t)-\tau \frac{\partial \mathbf{Q}(\varpi(t), t)}{\partial \varpi} \dot{\varpi}(t) \\
\\
+\frac{\tau^{2}}{2}\left[\frac{\partial \mathbf{Q}(\varpi(t), t)}{\partial \varpi} \ddot{\varpi}+\frac{\partial^{2} \mathbf{Q}(\varpi(t), t)}{\partial \varpi^{2}} \dot{\varpi}^{2}(t)\right]+\ldots
\end{aligned}
$$

Thus, it is natural to look at the case where $\mathbf{Q}$ depends on the price and its derivatives.

Finally, a natural extension is the case where $\mathbf{Q}$ has random fluctuations. This is particularly relevant if the energy production is subject to unpredictable changes - this is the case of wind energy. For the case where $\mathbf{Q}$ is random, we need to use the master equation as in [37].

\section{REFERENCES}

[1] N. Almulla, R. Ferreira, and D. Gomes. Two numerical approaches to stationary mean-field games. Dyn. Games Appl., 7(4):657-682, 2017.

[2] M. Bardi and I. Capuzzo-Dolcetta. Optimal control and viscosity solutions of HamiltonJacobi-Bellman equations. Birkhäuser Boston Inc., Boston, MA, 1997. With appendices by Maurizio Falcone and Pierpaolo Soravia.

[3] M. Burger, L. A. Caffarelli, P. A. Markowich, and M.-T. Wolfram. On the asymptotic behavior of a Boltzmann-type price formation model. Commun. Math. Sci., 12(7):1353-1361, 2014.

[4] M. Burger, L. A. Caffarelli, P. A. Markowich, and Marie-Therese Wolfram. On a Boltzmann-type price formation model. Proc. R. Soc. Lond. Ser. A Math. Phys. Eng. Sci., 469(2157):20130126, 20, 2013.

[5] L. A. Caffarelli, P. A. Markowich, and J.-F. Pietschmann. On a price formation free boundary model by Lasry and Lions. C. R. Math. Acad. Sci. Paris, 349(11-12):621-624, 2011.

[6] L. A. Caffarelli, P. A. Markowich, and M.-T. Wolfram. On a price formation free boundary model by Lasry and Lions: the Neumann problem. C. R. Math. Acad. Sci. Paris, 349(1516):841-844, 2011.

[7] F. Cagnetti, D. Gomes, H. Mitake, and H. V. Tran. A new method for large time behavior of degenerate viscous Hamilton-Jacobi equations with convex Hamiltonians. Ann. Inst. H. Poincaré Anal. Non Linéaire, 32(1):183-200, 2015.

[8] F. Cagnetti, D. Gomes, and H. V. Tran. Aubry-Mather measures in the nonconvex setting. SIAM J. Math. Anal., 43(6):2601-2629, 2011.

[9] P. Cardaliaguet. Notes on mean-field games. 2011.

[10] A. Clemence, B. Tahar Imen, and M. Anis. An Extended Mean Field Game for Storage in Smart Grids. ArXiv e-prints, October 2017.

[11] R. Couillet, S.M. Perlaza, H. Tembine, and M. Debbah. Electrical vehicles in the smart grid: A mean field game analysis. IEEE Journal on Selected Areas in Communications, 30(6):1086-1096, 2012. cited By 56.

[12] A. De Paola, D. Angeli, and G. Strbac. Distributed control of micro-storage devices with mean field games. IEEE Transactions on Smart Grid, 7(2):1119-1127, 2016.

[13] A. De Paola, V. Trovato, and D. Angeli. A mean field game approach for distributed control of thermostatic loads acting in simultaneous energy-frequency response markets. IEEE Transactions on Smart Grid, 2019.

[14] L. C. Evans. Partial Differential Equations. Graduate Studies in Mathematics. American Mathematical Society, 1998.

[15] L. C. Evans. Adjoint and compensated compactness methods for Hamilton-Jacobi PDE. Arch. Ration. Mech. Anal., 197(3):1053-1088, 2010. 
[16] L. C. Evans and D. Gomes. Effective Hamiltonians and averaging for Hamiltonian dynamics. I. Arch. Ration. Mech. Anal., 157(1):1-33, 2001.

[17] L. C. Evans and D. Gomes. Effective Hamiltonians and averaging for Hamiltonian dynamics II. Archive for rational mechanics and analysis, 161(4):271-305, 2002.

[18] W. H. Fleming and H. M. Soner. Controlled Markov processes and viscosity solutions, volume 25 of Stochastic Modelling and Applied Probability. Springer-Verlag, New York, 2006.

[19] D. Gomes, L. Lafleche, and L. Nurbekyan. A mean-field game economic growth model. Proceedings of the American Control Conference, 2016-July:4693-4698, 2016.

[20] D. Gomes, L. Nurbekyan, and E. Pimentel. Economic models and mean-field games theory. Publicações Matemáticas do IMPA. [IMPA Mathematical Publications]. Instituto Nacional de Matemática Pura e Aplicada (IMPA), Rio de Janeiro, 2015. 30o Colóquio Brasileiro de Matemática. [30th Brazilian Mathematics Colloquium].

[21] D. Gomes, E. Pimentel, and H. Sánchez-Morgado. Time-dependent mean-field games in the subquadratic case. Comm. Partial Differential Equations, 40(1):40-76, 2015.

[22] D. Gomes, E. Pimentel, and H. Sánchez-Morgado. Time-dependent mean-field games in the superquadratic case. ESAIM Control Optim. Calc. Var., 22(2):562-580, 2016.

[23] D. Gomes, E. Pimentel, and V. Voskanyan. Regularity theory for mean-field game systems. SpringerBriefs in Mathematics. Springer, [Cham], 2016.

[24] D. Gomes and J. Saúde. Mean field games models - a brief survey. Dyn. Games Appl., $4(2): 110-154,2014$.

[25] D. Gomes and J. Saude. Monotone numerical methods for finite-state mean-field games. arXiv preprint arXiv:1705.00174, 2017.

[26] J. Graber and C. Mouzouni. Variational mean field games for market competition. arXiv e-prints, page arXiv:1707.07853, Jul 2017.

[27] M. Huang, P. E. Caines, and R. P. Malhamé. Large-population cost-coupled LQG problems with nonuniform agents: individual-mass behavior and decentralized $\epsilon$-Nash equilibria. IEEE Trans. Automat. Control, 52(9):1560-1571, 2007.

[28] M. Huang, R. P. Malhamé, and P. E. Caines. Large population stochastic dynamic games: closed-loop McKean-Vlasov systems and the Nash certainty equivalence principle. Commun. Inf. Syst., 6(3):221-251, 2006.

[29] A.C. Kizilkale and R.P. Malhame. A class of collective target tracking problems in energy systems: Cooperative versus non-cooperative mean field control solutions. Proceedings of the IEEE Conference on Decision and Control, 2015-February(February):3493-3498, 2014.

[30] A.C. Kizilkale and R.P. Malhame. Collective target tracking mean field control for electric space heaters. 2014 22nd Mediterranean Conference on Control and Automation, MED 2014, pages 829-834, 2014.

[31] A.C. Kizilkale and R.P. Malhame. Collective target tracking mean field control for markovian jump-driven models of electric water heating loads. IFAC Proceedings Volumes (IFACPapersOnline), 19:1867-1872, 2014.

[32] A. Lachapelle, J.-M. Lasry, C.-A. Lehalle, and P.-L. Lions. Efficiency of the price formation process in presence of high frequency participants: a mean field game analysis. Mathematics and Financial Economics, 10(3):223-262, 2016.

[33] J.-M. Lasry and P.-L. Lions. Jeux à champ moyen. I. Le cas stationnaire. C. R. Math. Acad. Sci. Paris, 343(9):619-625, 2006.

[34] J.-M. Lasry and P.-L. Lions. Jeux à champ moyen. II. Horizon fini et contrôle optimal. C. R. Math. Acad. Sci. Paris, 343(10):679-684, 2006.

[35] J.-M. Lasry and P.-L. Lions. Mean field games. Jpn. J. Math., 2(1):229-260, 2007.

[36] J.-M. Lasry, P.-L. Lions, and O. Guéant. Mean field games and applications. Paris-Princeton lectures on Mathematical Finance, 2010.

[37] P. L. Lions. Collège de France course on mean-field games. 2007-2011.

[38] R. Malhamé and C.-Y. Chong. On the statistical properties of a cyclic diffusion process arising in the modeling of thermostat-controlled electric power system loads. SIAM J. Appl. Math., 48(2):465-480, 1988.

[39] R. Malhamé, S. Kamoun, and D. Dochain. On-line identification of electric load models for load management. In Advances in computing and control (Baton Rouge, LA, 1988), volume 130 of Lect. Notes Control Inf. Sci., pages 290-304. Springer, Berlin, 1989. 
[40] P. A. Markowich, N. Matevosyan, J.-F. Pietschmann, and M.-T. Wolfram. On a parabolic free boundary equation modeling price formation. Math. Models Methods Appl. Sci., 19(10):19291957, 2009.

(D. A. Gomes) King Abdullah University of Science and Technology (KAUST), CEMSE Division, Thuwal 23955-6900. Saudi Arabia.

Email address: diogo.gomes@kaust.edu.sa

(J. Saude) Carnegie Mellon University, Electrical and Computer Engineering Department. 5000 Forbes Avenue Pittsburgh, PA 15213-3890 USA.

Email address: jsaude@andrew.cmu.edu 